\title{
Predicting the vortex shedding frequency at the interface of the lateral cavities
}

\author{
Clément Perrot-Minot ${ }^{1, *}$, Emmanuel Mignot ${ }^{2}$, Nicolas Riviere $^{2}$, and Richard Perkins ${ }^{1}$ \\ ${ }^{1}$ Univ. Lyon, ECL Lyon, LMFA, CNRS, F-69130 Ecully, France \\ ${ }^{2}$ Univ. Lyon, INSA Lyon, LMFA, CNRS, F-69100 Villeurbanne, France
}

\begin{abstract}
The vortex shedding frequency in the mixing layer between a cavity and a main stream has been examined experimentally in absence of large oscillation of the free surface inside the cavity, called seiching. It was observed that the vortex shedding frequency follows a monotonically increasing trends with possible jumps from one to another with increasing Froude number of the main stream. These trends are obtained by solving a model based on the Rossiter approach. This model considers that the vortices shed in the mixing layer create some water depth variation at the impingement corner of the cavity. This water depth variation in turn generate pressure waves that propagates upstream and influence the vortex shedding process. Finally the measured vortex shedding frequencies correspond quite accurately to the frequencies predicted by the model. This highlights the existence of a resonant phenomena between vortices in the mixing layer of a lateral cavity and gravity waves even without any seiching phenomena.
\end{abstract}

\section{Introduction}

In both hydrology and acoustic fields, cavities are often encountered for industrial and environmental applications. Theses cavities can be defined as dead zones connected to a main stream. The large velocity gradient at the interface between the cavity and the main stream produces a mixing layer within which are observed spatially developing vortices. Theses vortices induce momentum exchange and generate recirculating cells in the cavity which size and number depend on the geometry of the cavity $[1,2]$. Moreover, the impact of these vortices on the downstream corner, after their advection along the mixing layer, induces waves that can trigger one dominant mode of the cavity. In aerodynamics problems, the flow velocity is high enough for the vortices to generate frequencies matching with the frequency of acoustic waves travelling within the cavity. In environmental problems, the velocity is smaller, and vortices can generate oscillation frequencies matching with the frequency of gravity waves travelling at the free-surface of the cavity. In case of matching, a resonant phenomena, called seiching, is likely to appear for specific hydrodynamic and geometric condition, inducing large free surface oscillation inside the cavity [3, 4].

The importance of vortices on the onset of gravity waves being assessed, it is necessary to explain their role and how they cause the resonance. The dynamics of vortices in lateral

\footnotetext{
*e-mail: clement.perrot-minot@insa-lyon.fr
} 
cavities has already been studied in the past [1-4]. At first sight, it is possible to consider that the mixing layer is subject to a convective instability, in fact a Kelvin-Helmholtz instability [5].

One main difference with classical K-H instabilities is the presence of the impingement and flow deviation on the downstream corner of the cavity. Wolfinger et al. [4] studied the vortex shedding frequency and observed that the frequency of vortices is constant along the mixing layer, which differs from free mixing layer [6]. Moreover, the evolution of the vortices frequency with the Froude number $\left(F_{r}=U / \sqrt{g h}\right.$ with $U$ the mean velocity inside the main channel, and $h$ the water depth at rest, see figure 1) exhibits two separate behaviours [4]. For low Froude number, typically $F_{r}<0.6$, the vortex shedding frequency increases linearly with increasing Froude numbers. For higher Froude numbers, $F_{r}>0.6$, the vortex shedding frequency remain constant, and equal to one of the natural frequencies of the cavity. This is the so-called lock-on stage, which produces the seiching phenomena.

The objective of the present paper is to explain the behaviour observed in absence of seiching,i.e. the phenomena responsible of the vortex shedding frequency and its presumed linear dependency of this frequency to the Froude number for $F_{r}<0.6$, and to propose a model to predict this frequency. A first part describes the experimental facility and measuring devices used to obtain the results shown in the second part. A third part is focused on the model used to predict the vortex shedding frequency.

\section{Experimental setup and process}

\subsection{Facilty}

The recirculating flow is generated by a submerged centrifugal pump, and its discharge is measured by an electromagnetic flow meter (ProMag50, Endress Hauser). The uncertainty of the flow meter is $\pm 0.05 \mathrm{~L}_{\mathrm{s}} \mathrm{s}^{-1}$. Water then flows into a $2 \mathrm{~m}$ long and $b=30 \mathrm{~cm}$ wide upstream glass channel. The flow then reaches the cavity. The cavity is made of a glass channel of similar dimension $(L=30 \mathrm{~cm})$, with a movable wall allowing to adapt the width of the cavity over the range $W=[0,200 \mathrm{~cm}]$. After the cavity, another $2 m$ long glass channel of similar dimension is employed before the downstream tank. Figure 1 provides a schematic view of the setup around the cavity with the notation and axes employed in this paper.

All experiments done in this project have been done under a strict protocol to insure independence of each measurement. For each tested configuration, the experimental conditions in the main stream are established while closing the cavity. Only when the water depth and mean velocity inside the main channel are perfectly stabilized, the cavity is suddenly opened. This protocol is used to get rid of the influence of the previous experiment on each new measure.

Several geometric and hydrodynamic conditions are tested and compared to the literature: a configuration with $W=50 \mathrm{~cm}$ and $h=5.8 \mathrm{~cm}$, then $W=60 \mathrm{~cm}$ and $h=5.8 \mathrm{~cm}$, and finally changing the water depth to obtain $W=60 \mathrm{~cm}$ and $h=4.8 \mathrm{~cm}$. The value chosen for $h$ insures to stay in the "deep" regime. The influence of the bottom wall friction can be estimated with the $S$ criteria introduced by Chu and Babarutsi [7] and further studied by Kolyshkin and Ghidaoui $[8,9]$. In our case, the order of magnitude of $S$ is around $10^{-3}$, while the critical $S_{c r}$ value determined by Kolyshkin and Ghidaoui [8] was $10^{-1}$.

The time evolution of water depth at the downstream corner of the cavity (view figure 1) is measured using an ultrasonic probe UNDK $20 \mathrm{I} 6912$ S35A from Baumer Electric AG. The sampling frequency is $200 \mathrm{~Hz}$ with a resolution inferior to $\Delta \approx 3 \mathrm{~mm}$.

\subsection{Velocity measurement}

Two kinds of velocity measurement are employed in this study. 


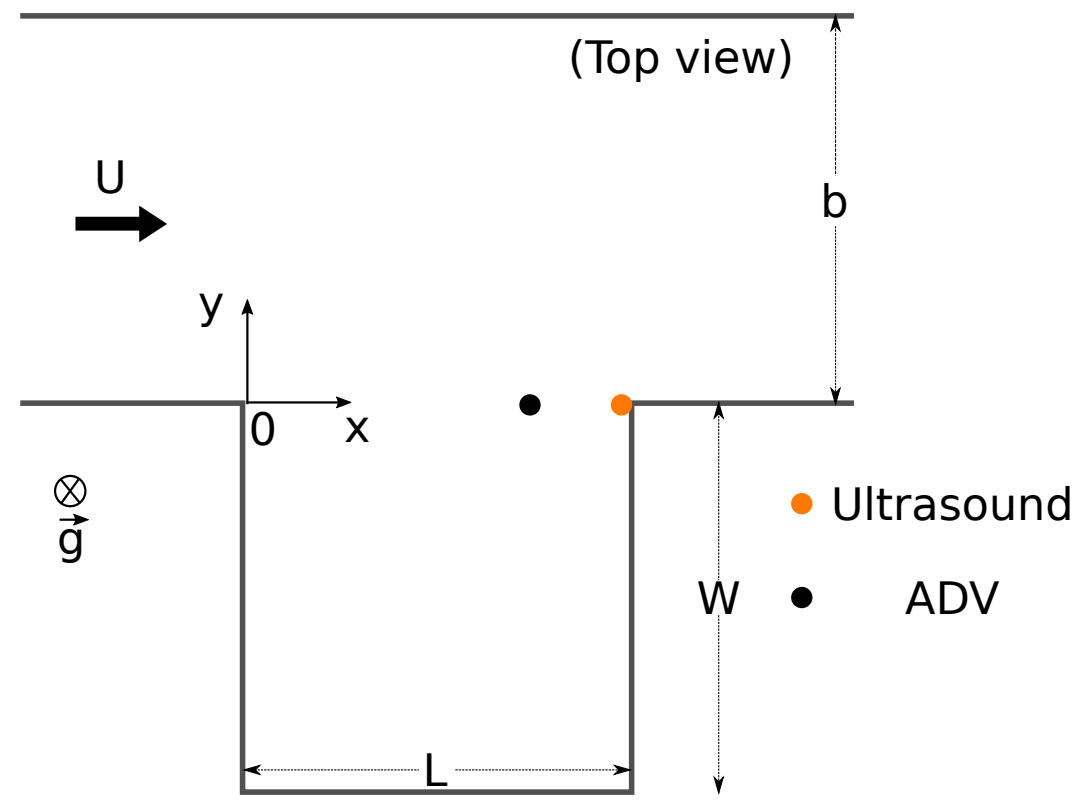

Figure 1. Schematic view of the lateral cavity and adjacent channel. Position of water depth measurement (orange dot) and transverse velocity measurement (black dot)

The Acoustic Doppler Velocimer (ADV) is employed to punctually measure the transverse component of the velocity at one location of the mixing layer, at an altitude of $z / h=0.8$. Measurements from both transverse velocity inside the mixing layer and water height at the impingement corner of the cavity give the same main frequency for every tested configuration and Froude number. For the rest of the paper, frequencies observed from ADV or ultrasonic probe will not be distinguished.

However, for some configurations, Particules Imagerie Velocimetry measurement is also used in order the visualize the vortices inside the mixing layer. The seeding is made with $50 \mu m$ hollow glass spheres. The laser sheet, created from the side of the channel, goes through the glass walls to en light a horizontal plane in the mixing at $z / h=0.8$. Finally, the images are taken from bellow, through a glass bottom wall, by AV Manta G-235B camera, 1936(H)x1216(V) pixels. Each set of PIV data is recorded at $70 \mathrm{fps}$ during 10 seconds. The PIV sets are used to track vortices and measure its spatial and temporal properties, such as wavelength $\lambda$, that correspond to the distance between two vortices, and the propagation speed of vortices $c_{\text {vortex }}$ in the mixing layer.

To identify the vortices, the critera $\gamma_{1}$ is employed. This criteria, defined by Graftetiaux et al. [10] and already applied to mixing layers by Mignot et al. [1] is mathematically written:

$$
\gamma_{1}(P)=\frac{1}{S} \int_{M \in S} \frac{(P M \wedge U(M)) \cdot z}{\|P M\| .\|U(M)\|} d S
$$

where $P$ is the point in space where $\gamma_{1}$ is calculated, $S$ is a circular surface in the plan of radius $R, M$ is a point in $S$ and $U(M)$ is the velocity at point $M$. This non-local criteria is only based on velocity direction (and not norm) and is equal to \pm 1 at vortices center, and 0 in pure strain zones. In order to further reduce the influence of stain in the deformation 


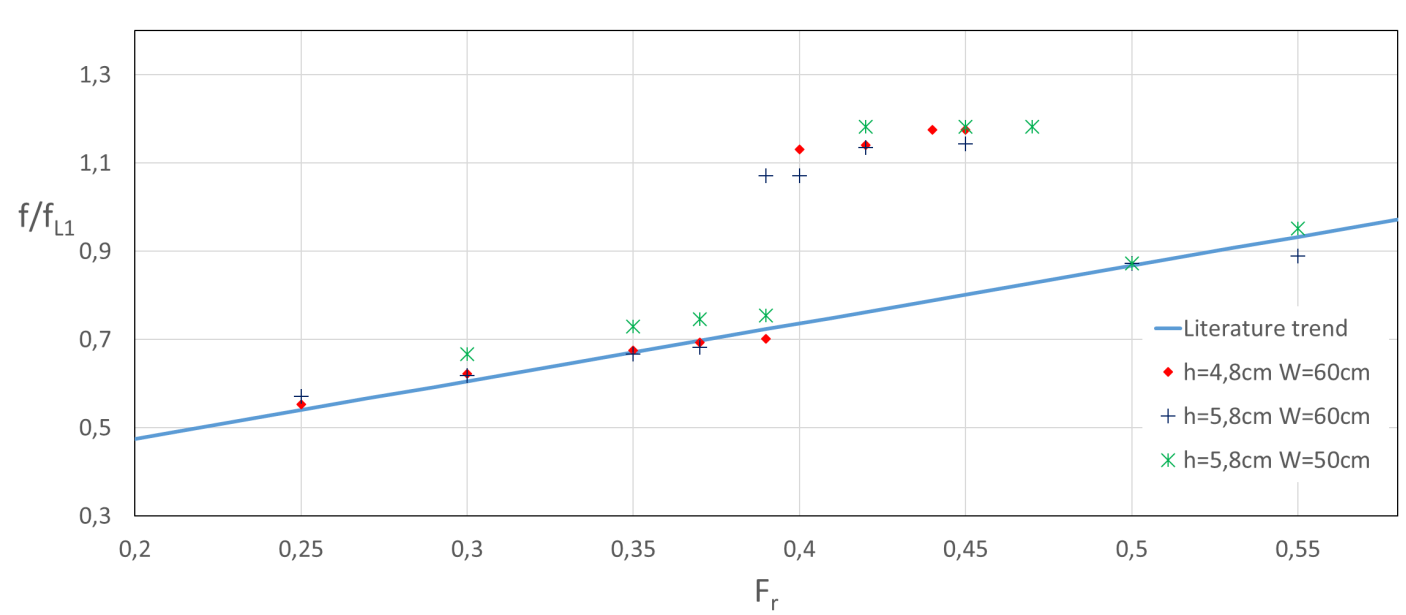

Figure 2. Evolution of characteristics frequency of mixing layer with Froude number. In blue, linear evolution of frequency acording to the literature [4]. Frequency jump at $F_{r}=0.4$ and $F_{r}=0.5$.

at the mixing layer, a Reynolds based decomposition have been employed, removing the time average velocity everywhere in the domain. Hence, one clockwise rotating vortex is artificially split into two counter rotating vortices in the fluctuating velocity field [1].

\section{Results}

Figure 2 shows the results from the three tested configurations. The characteristic frequency of the mixing layer $f$ normalized by the natural frequency of the cavity, $f_{L 1}=\sqrt{g h} / 2 L$, is plotted as a function of the Froude number of the main stream $\left(F_{r}=U / \sqrt{g h}\right), \sqrt{g h}$ being the velocity of pressure waves in the water.

It can also be further specified that the frequency derived from transverse velocity measurement is the same all along the cavity opening, as what have been found in the literature [4].

These results exhibit interesting behaviours. Every set of experiments done with different cavity depth and water height exhibits roughly the same results, so that the mixing layer behavior seems independent of $W$ and $h$. Furthermore, the frequencies measured seem to fit quite well the previous results of Wolfinger [4], represented by the solid line in figure 2 for Froude numbers in the interval $F_{r}=[0.25,0.4]$ and $F_{r}=[0.5,0.6]$. However, for Froude numbers $F_{r}=[0.4,0.5]$, the measured frequencies differ substantially from the Wolfinger et al.[4].

In the end, two frequency jump are observed at $F_{r}=0.4$ and $F_{r}=0.5$. These two jumps have not been observed in lateral cavity mixing layer with free surface in recent works. Nevertheless that this kind of jumps has been observed in the past in free surface problems when the impingement corner was moving, in particular by D.Rockwell [11].

To explain these jumps, PIV measurement are used to access the vortice velocities (noted $c_{v}$ ) and wavelength (noted $\lambda$ ) specifically around the frequency jumps. It is possible to see the moving vortices as a wave propagating downstream in the mixing layer. Hence we can relate its frequency $f$ to its velocity $c_{v}$ and its wavelength $\lambda: f=c_{v} / \lambda$. Figure 3 shows the time and space evolution of the center of vortices along the mixing layer. The wavelength 


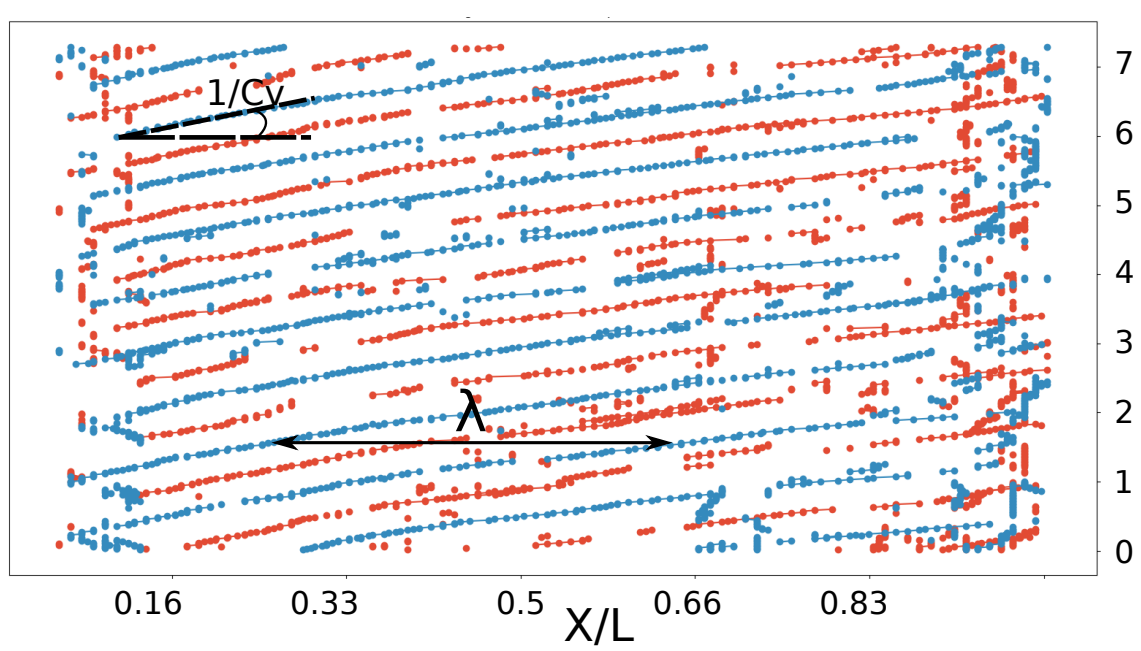

Time

7 (s)

6

5

4

3

2

1

0

Figure 3. Spatio-temporal displacement of vortices center. Blue dot correspond to the center of clockwise rotating vortices $\left(\gamma_{1}=-1\right)$ and red dot correspond to center of counter-clockwise rotating vortices $\left(\gamma_{1}=1\right)$. Wave length of vortices is the distance between two same color line at a fixed time. Celerity is inversely proportional to the slope of line

$\lambda$ corresponds to the distance between two consecutive vortices as shown in figure 3 (see Mignot et al. [1]). The celerity of vortices appears to be varying along the mixing layer, as the lines are not perfectly straight. The celerity of vortices increases almost linearly along the mixing layer, going from $c_{v} / U \approx 0.4$ at the upstream corner to $c_{v} / U \approx 0.65$ at the downstream corner, as we see the lines being less and less inclined along the mixing layer. We consider here the mean velocity along the mixing layer as the vortex celerity $c_{v}$.

Measurement reveals that the velocity of vortices in the mixing layer remains about constant as the frequency jumps occurs. Oppositely, the wavelength $\lambda$ of the instability exhibits a strong jump related to the frequency jump, with a value decreasing at the first frequency jump $\left(F_{r} \approx 0.4\right)$, and increasing at the second $\left(F_{r} \approx 0.5\right)$ (see figure 2$)$. This suggests that a mechanism forces the instability to adapt to a higher or lower frequency at a given Froude number, and that only discrete values of frequency and wavelength are allowed in this mechanism.

\section{Feedback Model}

The physical model proposed here is mainly used in acoustic cavities to explain the tones measured in the far field. The main idea comes from Powell et al.[12], and the mathematical version is known as the Rossiter model [13] that gives the Strouhal number $S_{t r}$ of a lateral rectangular cavity as:

$$
S_{t r}=\frac{n-\alpha}{c_{v}-M}
$$

with $n \in \mathbb{N}$ the mode $(1,2,3, \ldots), \alpha$ a empiric value often taken equal to 0.25 [13], $c_{v}$ the celerity of vortices and $M$ the Mach number $\left(M=U / U_{\text {pressure }}, U_{\text {pressure }}\right.$ being the pressure waves velocity and $U$ being the approaching flow velocity). No evident counterpart to this model is exist in environmental cavities. The physical model from which this formula comes from is fairly simple and is adapted here to the lateral cavity with free surface. 
Consider a vortex which has just formed near the upstream edge of the cavity. It will take a time $T_{1}$ to reach the downstream edge of the cavity, at an average velocity $c_{v}$, where:

$$
T_{1}=\frac{L}{c_{v}}
$$

Suppose that the impact of this vortex on the downstream corner leads to a pressure fluctuation which propagates back upstream towards the upstream corner; this perturbation travels at a speed $\sqrt{g h}-U_{i}$, with $U_{i}$ the averaged flow velocity at interface, so this feedback will take a time $T_{2}$ to reach the upstream corner, where:

$$
T_{2}=\frac{L}{\sqrt{g h}-U_{c}}
$$

For resonance, we can show that the condition is [14] :

$$
T_{1}+T_{2}=\frac{N}{f}
$$

where $N$ is an integer $(N=1,2,3, \ldots)$ and $f$ the peak frequency of the mixing layer.

Combining equations (3),(4) and (5) we obtain:

$$
\frac{N}{f}=\frac{L}{c_{v}}+\frac{L}{\sqrt{g h}-U_{i}}
$$

So that:

$$
f=F_{r} \frac{N}{L} \sqrt{g h} r_{v}\left[1-\frac{r_{v} F_{r}}{1+\left(r_{v}-r_{i}\right) F_{r}}\right]
$$

where $r_{v}=c_{v} / U$ and $r_{i}=U_{i} / U$. Both ratio are measured experimentally, with $r_{v} \in[0.5,0.6]$ and $r_{i} \approx 0.57$ for the three sets of experiments. The value of $r_{i}$ is in agreement with the literature $[15,16]$. The model is really sensitive to the value of $r_{v}$ but not to $r_{i}$.

Then we can make this equation dimensionless by dividing $f$ by the natural frequency $f_{L 1}$ :

$$
\frac{f}{f_{L 1}}=2 F_{r} N\left[\frac{r_{v}\left(1-r_{i} F_{r}\right)}{1+\left(r_{v}-r_{i}\right) F_{r}}\right]
$$

Solutions of (8) are plotted in figure 4 with the 3 first values of $N=1,2,3$. It is interesting to note that the Froude number appears naturally in the model, due to the influence of the main stream velocity $U$ and the natural frequency of the cavity dependency with pressure waves celerity $\sqrt{g h}$.

The model on figure 4 appears to fit quite well to the experimental data we acquired. This mechanism seems to be well suited to explain the discrete nature of the allowed frequencies in the mixing layer. We can see that for low Froude number (below $F_{r} \approx 0.4$ ), the dominant mode seems to be the mode $N=2$. After the frequency jump $\left(0.5>F_{r}>0.4\right)$, the dominant mode seem to be $N=3$ and finally for $F_{r}>0.5$, the dominant mode is again $N=2$. The physical meaning of the jumps, especially the one at $F_{r}=0.5$ is not captured by the model and remains unclear.

An important feature to notice here is the non-linear nature of the model that has been proposed. It differs from the linear increasing proposed by others authors (see [4] [3] and figure 2). 


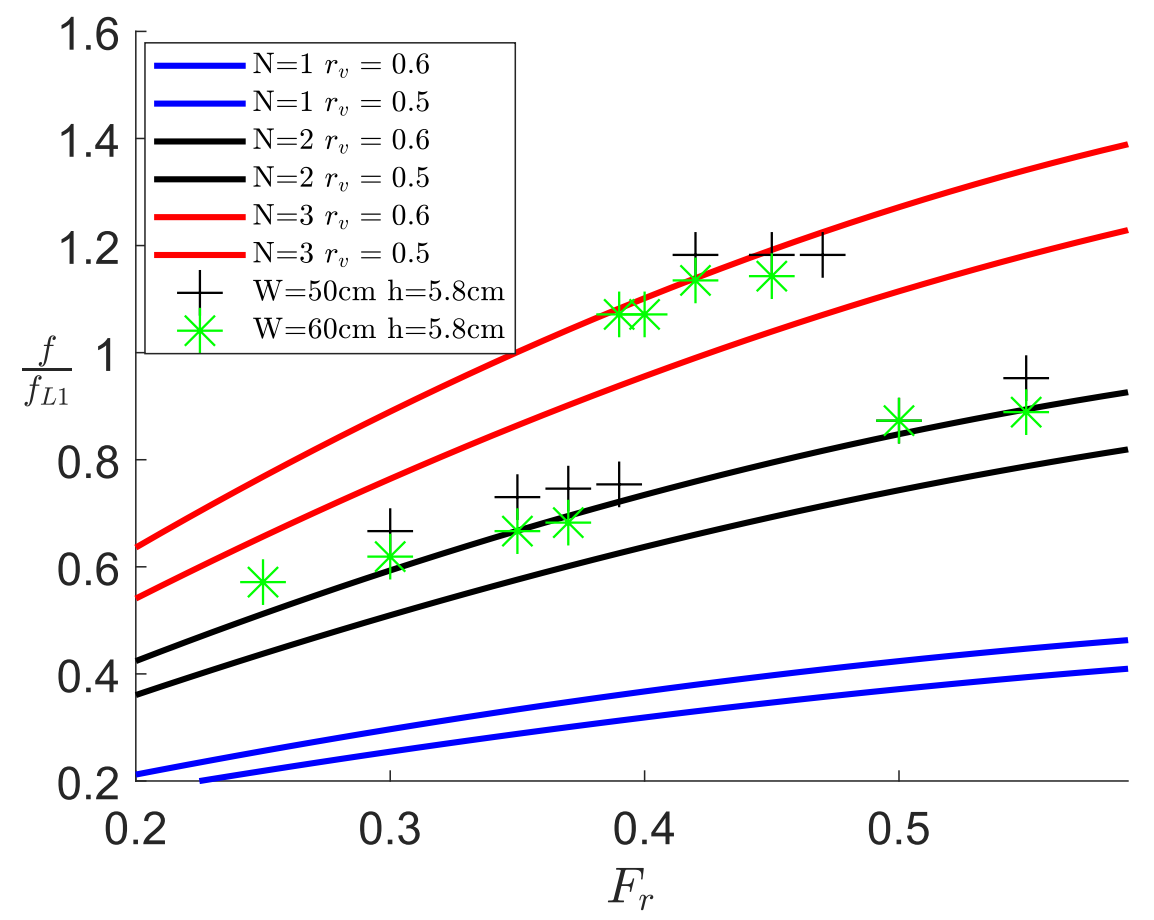

Figure 4. Feedback model (plain lines) plotted for the first 3 modes $N=1,2,3$ as a function of the Froude number with $r_{v}=0.5$ and 0.6$]$ and $r_{i}=0.57$. Two set of experimental datas are also plotted.

\section{Conclusion}

The behaviour of a mixing layer in a lateral cavity with free surface has been studied via velocity measurement. This study revealed that the behavior differs substantially from a free mixing layer. It has been observed that the vortex shedding frequency could only take discrete values for a given Froude number. Furthermore, the dependency of this discrete values with increasing Froude number has been found not to be linear as was suggested in previous works $[3,4]$

The phenomena associated with this behaviour has been described using a model based on a resonance between the vortices and the pressure waves created by the impact of the vortices on the impingement corner, propagating upstream. Previously, when studying such cavities, only one resonant phenomena was discussed, which was the seiching phenomena, appearing at high Froude number, typically $F_{r}>0.6$ and not considered in the present work. It has been shown in this work that another resonant phenomena actually occurs for lower Froude numbers and is responsible for the selection of vortex shedding frequency in the mixing layer. In fact, the matching between the vortex shedding frequency and the natural frequency of cavity (seiching) is the matching of two resonant phenomena involving pressure waves. This consideration may modify the approach used to study the seiching phenomena in future works.

The feedback process described herein is however unable to determine which of the discrete frequency will be present for a given Froude number and thus unables us to predict the Froude numbers at which jumps are observed in the measured curves. Linear spatial stability, 
as well as influence of natural cavity modes for high Froude number, will now be applied to intent to explain this selection process.

\section{References}

[1] E.Mignot, W.Cai, G.Launay, N.Riviere, C.Escauriazia, Phycis of Fluids 28 (2016)

[2] W. Cai, Ph.D. thesis, LMFA, INSA Lyon (2016)

[3] B. Tuna, E.Tinar, D.Rockwell, Experiments in Fluids 54 (2013)

[4] M.Wolfinger, C. Ozen, D.Rockwell, Physics of Fluids 24 (2012)

[5] S.Bien, J.F.Driscoll, B.R.Elbing, Experiments in Fluids 51, 51 (2010)

[6] C.Godrèche, P.Manneville, B.Castaing, P.Huerre, M.Rossi, V.Hakim, S.Fauve, G.Joulin, P.Vidal, Hydrodynamics and Nonlinear Instabilities (Cambridge University Press, 1998)

[7] V. Chu, S.Babarutsi, Journal of Hydraulic Engineering 114, 1257 (1988)

[8] A. Kolyshkin, M. Ghidaoui, Journal of Hydraulic Engineering 128, 1076 (2002)

[9] M. Ghidaoui, A. Kolyshkin, Journal of Hydraulic Engineering 548, 309 (2006)

[10] L. Graftieaux, M. Michard, N. Grosjean, Measurement Science and Technology 12, 1422 (2001)

[11] C.Knisely, D.Rockwell, Journal of Fluid Mechanics 116, 157 (1982)

[12] A. Powell, The journal of the Acoustical Society of America 33 (1961)

[13] J.Rossiter, Aeronautical Research Council Reports and Memoranda 3438 (1964)

[14] C. Ho, N.S.Nosseir, Journal of Fluid Mechanics 105, 119 (1981)

[15] E.Mingot, W.Cai, J. Polanco, C.Escauriaza, N.Riviere, Environnemental Fluid Mechanics 17, 429-448 (2017)

[16] D.F.Hill, Journal of Hydraulic Engineering 140 (2013) 